\title{
Acute Intermittent Porphyria with SIADH and Fluctuating Dysautonomia
}

\author{
Nabin A, ${ }^{1}$ LJ Thapa, ${ }^{2}$ Paudel $R,{ }^{3}$ Rana PVS ${ }^{4}$
}

${ }^{1}$ Manipal Teaching Hospital,

Manipal College of Medical Sciences, Pokhara.

${ }^{2}$ Department of Medicine

$3,{ }^{4}$ Department of Medicine \& Neurology, College of Medical Sciences, Bharatpur (Chitwan)

\section{Corresponding Author}

PVS Rana

Department of Medicine \& Neurology

College of Medical Sciences, Bharatpur (Nepal)

Email: rananirmalpvs @ gmail.com.

\section{Citation}

Nabin A, LJ Thapa, Paudel R, Rana PVS. Acute Intermittent Porphyria with SIADH and Fluctuating Dysautonomia. Kathmandu Univ Med J 2012;38(2):96-99.

\begin{abstract}
Three cases of acute intermittent porphyria are reported. While in first case severe pain in abdomen with intermittent exacerbation was the only presentation, the second patient presented as accelerated hypertension and acute abdominal crises in whom the clinical course was characterized by development of deep coma due to inappropriate secretion of antidiuretic hormone before she made complete recovery. The third patient, initially manifested as acute encephalitic syndrome. After initial improvement, she developed features of acute intermittent porphyria i.e. acute abdomen, neuropsychiatric symptoms, and rapidly progressing acute motor neuropathy leading to respiratory and bulbar paralysis. In addition, she developed severe and fluctuating dysautonomia leading to cardiac arrest and fatal termination. The importance of early diagnosis, recognition of autonomic disturbances, prompt treatment and counseling for avoidance of precipitating factors is stressed.
\end{abstract}

\section{KEY WORDS}

Acute intermittent porphyria (AIP), Autonomic neuropathy, Dysautonomia, $d$-aminolevulinic acid (d-ALA)' Inappropriate secretion of antiduretic hormone (SIADH, Hematin, Porphobilinogen (PBG)

\section{INTRODUCTION}

Porphyria is a rare metabolic disorder having autosomal dominant inheritance with incomplete penetrance with some cases having autosomal recessive and complex inheritance..$^{1,2,3}$ The gene coding for enzymes has been characterized and several mutation in porphyria genes are reported. ${ }^{1}$ Though genetically heterogenous, different porphyrias have a uniform clinical phenotype of the three hepatic porphyrias having acute presentation, acute intermittent porphyria (AIP) is commonest and usually manifest after puberty predominantly affecting young female population. ${ }^{1,4}$ It present with acute abdominal crisis and neuropsychiatric manifestations. When inadvertent administration of precipitating factors continues in misdiagnosed cases, a rapidly progressing sensorimotor neuropathy develops with respiratory and bulbar paralysis and dysautonomia. Advancement in ICU care, avoidance of precipitating factors and prompt treatment with haematin, has reduced the mortality from about $30 \%$ to less than $10 \% .^{5,6}$ In addition these patients need constant follow up for early detection of hepatoma, hypertension and renal impairment. ${ }^{7,8}$ and modifications of treatment of subsequent systemic diseases developing in them. Three cases of intermittent porphyria, diagnosed and treated are reported. Importance of recognition of syndrome of inappropriate antidiuretic hormone (SIADH) and potentially fatal dysautonomia is stressed and literature is reviewed.

\section{CASE REPORT}

Case 1: MS (HN 17023), a 20 years old lady, was admitted with severe generalized abdominal pain and vomiting She gave history of similar episode, but of lesser severity, in the past. At admission she was afebrile with pulse 96/ min, Respiration-20/min and BP $1140 / 90$ mms of Hg. Per abdominal examination revealed diffuse tenderness. No lump, rigidity, guarding or free fluid detected. Rest systems were normal. Investigations i.e. complete hemogram, urinalysis, serum electrolytes, renal and metabolic parameters, ECG, USG abdomen were normal. She was 
initially treated as acute pancreatitis. However, detection of high colored which changed color on exposure to sunlight, porphyria was suspected and later confirmed by qualitative screening of urine for PBG. She was treated with glucose

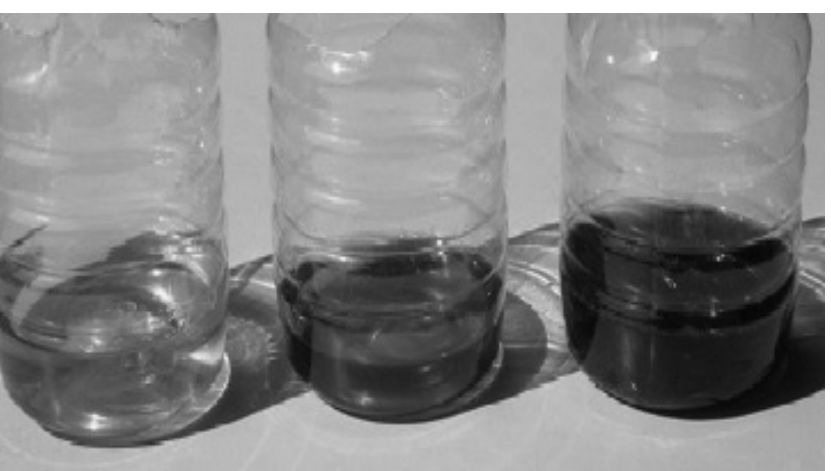

Figure 1(a). Case-2:Change of urine color on exposure to sun (Case 1).

administration, antispasmodics and chlorpromazine and made rapid and full recovery.

Case 2: AG (HN: 214954), a 25 years old lady presented with severe and continuous upper abdominal pain of five days duration. It was not associated with vomiting, bowel disturbances or genitourinary symptoms. However, she gave h/o of abdominal pain in the past lasting for variable period. At admission she was in agony and was afebrile with pulse 68/min, BP $160 / 130 \mathrm{~mm}$ of $\mathrm{Hg}$. Abdominal examination revealed soft abdomen with diffuse tenderness. Bowel sounds were sluggish. No organomegaly, rigidity, free fluid detected. Rest systems were normal. She was initially diagnosed and treated as acute pancreatic. Investigation revealed blood urea $56 \mathrm{mg} /$ $\mathrm{dl}$ and serum creatinine $1.7 \mathrm{mg} / \mathrm{dl}$. Other investigations i.e. including hemogram, urine analysis, blood sugar, serum electrolytes, liver function tests, serum amylase, ECG, X-ray chest \& abdomen, USG abdomen, upper GI endoscopy and urinary catecholamine assay were normal. Subsequently, in view of accelerated hypertension, tachycardia possibility of porphyria was suspected and diagnosis confirmed by

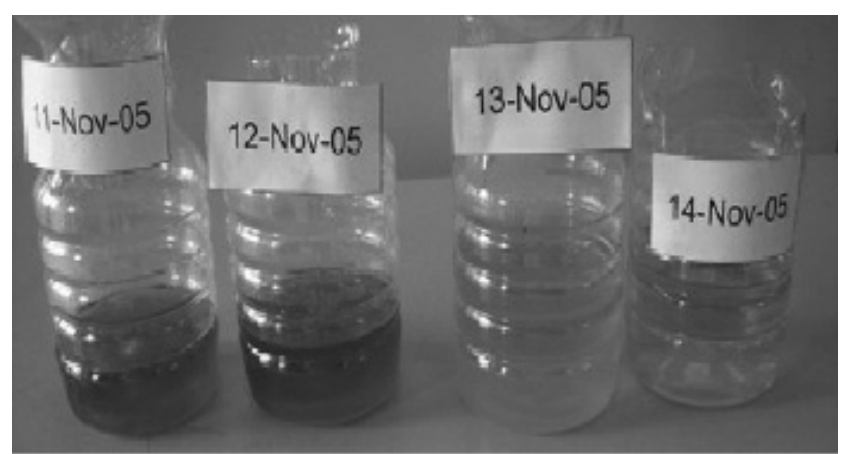

Figure 1(b). Case-2: Gradual Clearing of urine with recovery.

noting change of color of urine on exposure to sunlight (fig$1(b))$ and qualitative test for porphobilogen.

She was treated with high parenteral carbohydrate intake, antihypertensive agent, chlorpromazine, pethidine and antispasmodics. She made gradual recovery with normalization of BP and marked reduction of abdominal symptoms. However, on day 8thth of her admission she became drowsy, incontinent and lapsed into deep coma. In view of normalization of blood pressure, passing of normal colored urine, absence of localizing neurological deficits and normal pupils, possibility of SIADH suspected and confirmed. With continuation of high parenteral dextrose and restriction of fluid intake, she recovered gradually and was discharged on 17th day with advice to avoid precipitating factors.

Case 3: HT (HN:215841), a 17 years old girl was admitted with acute neurological illness manifesting with fever, higher function changes and recurrent seizures. At admission she was febrile (Temp-99.6 ${ }^{\circ} \mathrm{F}$ ) with pulse-102/ min and BP 160/ $110 \mathrm{~mm} \mathrm{Hg}$. She was responding to painful stimuli by localization. No lateralizing neurological deficits detected. Deep tendon reflexes were symmetrical with plantar response mute on both sides. Pupils were equal and reacting to light normally. Fundus examination was normal. No neck rigidity was detected. Rest systems were normal. She was diagnosed as a case of meningoencephalitis of probable viral etiology with herpes simplex encephalitis as the likely possibility. Investigations done i.e. Blood: Hb 14.8 gm/dl, TLC 14800/cmm, DLC P 89\%, L19\%; Serum electrolytes (mmol/L) : $\mathrm{Na}^{+} 104, \mathrm{~K}^{+} 3.6$; CT Scan revealed small pinched ventricles with a hydpodense non enhancing lesion in right temporal region; CSF examination revealed normal biochemistry. Cell: WBC 6/cmms and RBC $1200 / \mathrm{cmm}$. Blood sugar, renal parameters, Liver function tests, ECG and X-Ray chest were normal. EEG showed no periodic discharges. Her seizures were controlled with IV diazepam and diphenyl hydantoin (DPH). She was treated with acyclovir, IV antibiotics, antihypertensive, antipyretics, IV alimentation and sodium supplementation. Maintenance dose of DPH continued. On day $7^{\text {th }}$ of her admission, she was afebrile, had normal orientation and was taking fluid orally. She was extubated, transferred out of ICU and ambulation started. A day later, she was noted to be passing high colored urine which changed color on exposure to sun suggesting porphyria (confirmed by qualitative test for porphobilinogen). DPH was withdrawn and replaced with gabapantine and high dextrose alimentation started.

However, on day $11^{\text {th }}$ she developed abnormal behavior, tachycardia, and tachypnoea with spO2 falling to $70 \%$ and rapidly progressing areflexic quadriparesis, bulbar and respiratory paralysis. She was put on ventilator and DVT prophylaxis started. Other treatment continued. On day $15^{\text {th }}$, fluctuating BP (150-160/90-110 to $70-80 / 45-50 \mathrm{mms}$ of $\mathrm{Hg}$ ) and tachycardia, requiring frequent modification of antihypertensive drugs doses. Her BP stabilized but tachycardia persisted. There was no improvement in neuropathy. Two days later, she suffered a genralized seizure (controlled with diazepam) and developed acute abdominal pain and features of paralytic ileus. QTC prolongation was noted on day $18^{\text {th }}$. She continued to have frequent cardiac arrhythmia. Inspite of continued intensive care, her illness terminated fatally when she had cardiac 
arrest following ventricular tachycardia.

\section{DISCUSSION}

Due to varied symptomatology AIP is often misdiagnosed as is evident by the cases referred above. ${ }^{9}$ While case 1 and 2 had the referral diagnosis of acute pancreatitis, the third case developed symptoms of acute porphyria while recovering from encephalitic illness. In addition in case -2 an alternative diagnosis $s$ of pheochromocytoma and polyarteritis nodosa was considered and excluded. Clinical presentation in the cases presented was dominated by dysautonomia i.e. persistent tachycardia and mild hypertension (Case-1), accelerated hypertension and tachycardia (Case-2) and persistent tachycardia and hypertension followed by episodes of hyper and hypotension, tachycardia and bradycardia and cardiac arrhythmias respectively.

In AIP, neurotoxic d-aminolaevulinic acid and porphobilinogen accumulates due to induction of haem synthesis and their subsequent excretion from liver in large amount. ${ }^{10}$ The areas lacking barrier protection i.e. peripheral and autonomic nervous system and hypothalamic and limbic areas of brain are affected. ${ }^{10} \mathrm{~A}$ high incidence of symptoms attributable to autonomic neuropathy also suggest high vulnerability of autonomic nerves to abnormal accumulation of porphyrin metabolites. ${ }^{11,12}$ Peripheral neuropathy in porphyria is mainly an axonal neuropathy which is followed by "dying back" degeneration Tachycardia is attributable to unopposed sympathetic activity due to vagal damage and hypertension is the result of deafferentation of baroreceptors due to damage to vagus and glossopharyngeal nerves, Rana et al., (1981) studied five cases of porphyria when they were recovering. ${ }^{11-14,17}$ In addition to acute abdominal pain, the common combination was of hypertension, tachycardia and abnormal sweating patterns and diarrhea was noted 3 cases. Postural hypotension was noted in 2 patients with one having postural symptoms. Non invasive tests for ANS functions revealed abnormal valsalva ratio $(1.01+/-0.05)$ and $\mathrm{E}$ : I ratio $(1.00+/-0.16)$ as compared to $1.41+/-0.35$ and $1.25+/-0.30$ in control groups. Cold immersion test and response to atropine and propranolol was abnormal in 4, 4 and 3 patients respectively. Other studies also noted dysautonomia using similar noninvasive tests. ${ }^{18,19,20}$ Focal vascular damage with reversible cerebral edema has been reported in patients presenting as encephalopathy. ${ }^{15}$ These patients also show high catecholamines in urine due to inhibition of its uptake by platelets by raised d-ALA and porphobilinogen that may be confused with pheochromocytoma. However, urine catecholamines were normal in Case -2 were this diagnosis was considered. ${ }^{16}$

Motor neuropathy and dysautonomia of porphyria resemble AIDP. Loss of reflexes corresponding to muscle weakness, severe abdominal pain, neuropsychiatric symptoms and occurrence of seizures differentiate porphyria from AIDP.
Early proximal muscles involvement differentiates this from other toxins induced dying back neuropathy. Also, combination of focal CNS involvement and peripheral neuropathy is unusual for other neuropathies. Heavy metal poisoning (i.e. arsenic \& lead and thallium) presents with abdominal pain, seizures and encepahopathy and simulates AIP making screening for heavy metals a mandatory requirement.

To avoid misdiagnosis and potential fatality in acute porphyria, suspected patients should be investigated to confirm the diagnosis. ${ }^{21}$ Where qualitative and quatitative estimation of urinary PBG is not possible a simple test of observing urine color after exposure to sunlight is enough as was done in present cases. AIP is confirmed by marked rise in urinary porphobilinogen (uroporphyrin 1 \& III more than coproporphyrin 1 \& III), fecal corpoporhyrins normal or slightly raised and corpoporphyrin III/I ratio less than 2. ${ }^{21}$ If all three are normal porphyria is excluded. If only erythrocyte PBG deaminase activity is abnormal, analysis of HMBS gene is needed. If positive it suggests, AIP in remission or in latent stage when family history is positive.

Specific treatment of AIP administration of haem preparation (i.e. Hematin argenate or hematin) It should be administered at an earliest.The dose is $3 \mathrm{mg} / \mathrm{kg} /$ day for 3-4 consecutive days Hematin was not available for treatment. ${ }^{22}$ Treatment in case 1 and 2 case consisted of parenteral high carbohydrate intake, antispasmodics, diazepam and atenolol. While in first case complete remission was obtained, in case- 2 abdominal pain was relieved on day 5th day but she developed altered sensorium leading to deep coma. SIADH was suspected and confirmed as the cause of coma. It responded to fluid restriction. In Case-3, case diagnosis of encephalitis was entertained as fever is not a feature of acute porphyria. It was supported by improvement in neurological status inspite of administration of DPH. Later, she developed severe degree of dysautonomia with changing profile. It is postulated that she had AIP in latent stage when admitted which manifested with continued administration of DPH.

Complete recovery in second case inspite of prolong coma emphasize the importance of early recognition and prompt treatment of SIADH, an adverse prognostic factor. Development of acute neuropathy with bulbar and respiratory paralysis requiring mechanical ventilation and severe fluctuating dysautonomia are associated with high mortality. ${ }^{23}$ Dysautonomia manifesting with fluctuating BP , episodic bradycardia followed by tachycardia and, ventricular fibrillation was the cause of fatal termination in third case.

As development of complication can not be predicted, all AIP cases should be treated in ICU for constant monitoring for dysautonomia and for institution of appropriate remedial measures similar to that applied for AIDP cases, where similar dysautonomia is frequently noted. ${ }^{24}$ Dysautonomia persist even in latent phase suggesting 
subclinical neuropathy in porphyria. ${ }^{17}$ Continued monitoring of autonomic functions should be continued even when patients have recovered from acute episode.

\section{REFERENCES}

1. Anderson KE, Sassa S, Bishop DF, Desnick RJ. Disorders of hem biosynthesis: X-linked sideroblastic anemia and the porphyrias. In Scriver CR, Beaudet A, Sly WS, Valle D (Eds). The metabolic and molecular basis of inherited disease.8th Edition. New York: McGraw Hill, 2001:2991-3062.

2. Von und Zu Frauberg M, Pischik E, Udd L, Kauppinen R. Clinical and biochemical characteristics and genotype phenotype correlation in 143 Finish and Russian patients with acute intermittent porphyria. Medicine [Baltimore]. 2005; 84: 35-47. Kauppinen R. Porphyrias. Seminar. Lancet. 2005; 365: 241-252.

3. Moore MR, McColl KE, Rimingto C, Goldberg A. Disorders of Porphyrin metabolism. New York: Plenum Publishing Corporation; 1987.

4. Andersson C, Innala E, Backstrom T. Acute intermittent porphyria in women: Clinical expression, use and experience of exogenous sex hormones - a population based study in Sweeden. J Intern Med. 2003; 254: 176-83.

5. Goldberg, A. Acute intermittent porphyria. A study of 59 cases. Quart J Med. 1959; 28: 183-209.

6. Stein, JA, Tschudy DP. Acute intermittent porphyria: a clinical and biochemical study of 46 patients. Medicine. 1970; 49: 1-16.

7. Kauppinen R, Mustajoki, P. Acute hepatic porphyria and hepatocellular carcinoma. Br J cancer. 1988; 57;117-120.

8. Anderson C, Lithner F. Hypertension and renal disease in patients with acute intermittent porphyria. J Int Med. 1994; 236: 169-175.

9. Crimlisk $\mathrm{H}$. The little imitator porphyria. A neuropsychiatric disorder. J Neurol Neurosurg Psychiat. 1997; 62: 319-28.

10. Meyer UA, Schuurmanns MM, Lindberg RL. Acute Porphyrias: Pathogenesis of neurological manifestations. Seminar Liver Dis. 1998; 18: 43-52.

11. Ridley A, Heirons R, Cavanagh JB. Tachycardia and neuropathy in of porphyria. Lancet II.1969; 708-710.

12. Gorchein A. Autonomic neuropathy in Porphyria. In Bannister R (Ed) Autonomic failure; A Text Book of Clinical Disorders of Autonomic Nervous System. Edition(2).New York, Tokyo: Oxford University Press, Oxford ;1988, Chapter 40, pp 215-232.
13. Gibson KB, Goldenberg JA. The neuropathology of acute porphyria. J Pathol Bacteriol. 1956; 71: 495-509

14. Windebank AJ, Bonkovsky HL. Porphyric neuropathy. In Dyck, AJ, Thomas PK (Eds). Peripheral Neuropathy Vol II Third Edition. WB Saunders Co. Philadelphia: 150; 1469-1474.

15. Yen PS, Chen CI, Lui CC, Wai YY, Wan YL. Diffussion weighted magnetic resonance imaging pophyric encephalopathy : a case report. Eur Neurol. 2002;48: 119-121.

16. Beal MF, Atuk NO, Wesfall TC. Catecholamine uptake, accumulation and release in acute porpyria. J Clin Invest. 1977; 60:1141-114815. Crimlisk HL. The little imitator porphyria: a neuropsychiatric syndrome. J Nnurol Neurosurg Psychiat. 1997; 62: 319-328.

17. Rana PVS, Suri ML, Sahai B. A study of autonomic functions in neurological disorders. AFRC Project No 1030/78.

18. Gupta GL, Saksena HL, Gupta BD. Cardiac dysautonomia in acute intermittent porphyria. Ind J Med Res. 1983 (a); 78: 253-256.

19. Gupta GL, Saksena HL, Gupta BD. Acute neuropathy and hypertension in porphyria. J Ass Phy India. 1983 (b); 31: 359.

20. Yeung Laiwah AAC, Mac Phee GJA, Boyle P. Autonomic neuropathy in acute intermittent porphyria. J Neurol Neurosurg Psychiat. 1985; 48: $1025-1030$.

21. De Rooji WM, Edixhoven A, Wilson, H. Porphyria: a diagnostic approach. In Kadish KM, Smith KM, Smith R (Esds). The porphyrin handbook. St Luis Elsevier. 2003; 211-245.

22. Mustajoki $P$, Nordman Y. Early administration of heme arginate for acute porphyria attacks. Arch Intern Med. 1993; 153: 2004-208.

23. Kuppinen R, Mustajoki P. Prognosis of acute porphyria: occurrence of acute attack , precipitating factors and associated diseases. Medicine 1992; 71: 1-13.

24. Rana PVS. Autonomic dysfunctions in Guillain Barre Syndrome. In Sinha KK, Chandra P (Eds) Progress in Neurosciences. Published by Neurological Society of India.Vol II; 1990, 157-165. 